\title{
Long-term outcome of patients with urea cycle disorders and the question of neonatal screening
}

Published online: 21 November 2003

(c) Springer-Verlag 2003

\begin{abstract}
With regard to the principles established for neonatal population screening, the question arises whether for patients with urea cycle disorders there is an accepted treatment which really affects the disease course and prognosis as compared to the natural history of these diseases. A retrospective study of 88 patients was performed. Based on questionnaires, the survival rate and neurodevelopmental outcome of patients treated with protein restriction alone was compared to the new therapy introduced in the 1980s with added citrulline/arginine, essential amino acids for improving the amino acid composition of the restricted natural protein and benzoate. Survival of patients with neonatal presentation was improved by the extensive therapy but this mostly at the cost of an increasing number of retarded patients. Long-term outcome did not differ significantly between the two treatments. Of all patients, $56 \%$ were symptomatic within 4 days of age and $67 \%$ within the 1st week. Thus a prevention of irreversible damage by neonatal screening on blood obtained at 3-4 days of life is questionable. Whether the benefit of obtaining a rapid diagnosis, e.g. for allowing proper counselling and prospective treatment, is acceptable for the parents of prospective patients remains open. The organisation of a dense network of specialised metabolic centres with sufficient staff and resources is a prior condition for any screening programme in order to ensure the rapid diagnosis, follow-up of treatment and counselling of a cumulative number of affected chronic patients needing this support. A commitment on a long-term basis by the institutions is needed in view of the health budget restrictions. Conclusion: In the short term, the goal is to detect hyperammonaemic patients as early as possible
\end{abstract}

C. Bachmann

Laboratoire Central de Chimie Clinique, Centre Hospitalier Universitaire Vaudois, Bugnon 46, 1011 Lausanne, Switzerland E-mail: Claude. Bachmann@chuv.hospvd.ch Tel.: + 41-21-3144144

Fax: +41-21-3144133 with special emphasis on sick neonates. In practice, quantitative plasma ammonia determination without delay is recommended in any newborn for whom a sepsis work-up is considered and in children who refuse feeding or vomit and show alterations of consciousness and/ or neurological symptoms.

Keywords Hyperammonaemia - Inborn errors of metabolism $\cdot$ Neonatal screening $\cdot$ Outcome $\cdot$ Urea cycle disorders

Abbreviations $P K U$ phenylketonuria $U C D$ urea cycle disorder

\section{Introduction}

The opportunity of using new techniques in neonatal screening for genetic metabolic disorders has led to suggestions to include urea cycle disorders (UCD) in such programmes. The success of neonatal screening for phenylketonuria (PKU) has stimulated scientists to adapt and develop methods for testing markers for other metabolic disorders and start pilot programmes in order to widen the range of analytes tested in neonatal screening. Whilst showing the analytical feasibility, some have assumed implicitly that other diseases fulfill the same basic criteria as PKU and that feasibility is the main hurdle for introducing new programmes. However, past experience should be taken into account and accepted criteria, described in (still valid!) agreements between governments, should be respected.

\section{Background}

The conditions to be fulfilled by population screening programmes had been initially phrased by Wilson and Jungner [15] followed by practical proposals by an expert group of the WHO [3]. When in 1979 a controversy arose concerning new disease groups, the Public Health 
Committee of the European Council updated the recommendations of 1972. This led to a report on "Neonatal Mass Screening for Metabolic Disorders" which was adopted by the Public Health Committee (parties: Belgium, France, Federal Republic of Germany, Italy, Luxembourg, the Netherlands, the United Kingdom; Austria, Denmark, Ireland and Switzerland). A short version was published [4]. The introduction of tandem mass spectrometric techniques into clinical laboratories incited the British Health Technology Assessment Groups to commission an expert group to analyse the cost, yield and outcome in neonatal screening for inborn errors of metabolism (including psychological and ethical issues). This analysis is an excellent summary and a thorough and careful discussion on the many aspects of neonatal screening [10].

Concerning the principles of early disease detection in UCD some questions still remain unanswered:

1. Is there an accepted treatment for patients with recognised disease which really affects the disease course and prognosis as compared to the natural history of UCD? Are long-term side-effects of treatment known? ${ }^{1}$

2. Are enough facilities available for rapid diagnosis and treatment in order to ensure adequate control and adaptation of therapy without excessive travel distance for the patients? Is there a commitment for guaranteeing the resources needed (specialised knowhow and equipment) on a long-term basis?

3 Which test would be suitable for recognising all the UCD at an early or even latent stage (very high sensitivity, with tolerable false-positive rates)?

4. Would the screening and its consequences be acceptable to the parents (and prospective patients) after adequate information?

When confronted with hyperammonaemic patients (especially neonates) who are in coma, one has to decide either on palliative or intensive treatment and the question of the individual prognosis arises in the discussion with the parents. There are some data from large centres, mostly concerning survival rates or restricted to patients having survived the neonatal period [5]. Msall et al. [9] and Uchino et al. [13] addressed the question of neurodevelopmental handicap; but to our knowledge there are no data comparing the outcome of patients treated with the actual means ("new therapy") as compared to the natural history of the UCD ("old therapy") for testing the improvement. We consider the "old therapy" with protein restriction (and some acute interventions of low efficiency like blood exchange transfusion and peritoneal dialysis) as being close to the natural history of the UCD with respect to long-term outcome. The "new therapy" with

\footnotetext{
${ }^{1}$ It is still unknown if the amenorrhoea/menstrual dysfunction which occurred in $23 \%$ of the menstruating patients treated with phenylbutyrate as reported by Wiech et al. [14] was due to this drug or to other causes like inadequate protein supply.
}

supplements of missing urea cycle products (arginine or citrulline), optimised nitrogen supply by combining protein restriction with added essential amino acids and drug-induced diversion of excess nitrogen to alternate pathways (e.g. benzoate) has been used since the early 1980s. By comparing the long-term outcome of patients (not prospectively treated) with "new" and "old" therapy, we asked the following question: does the "new" treatment of the symptomatic clinical condition affect its course and prognosis with respect to life expectancy and more importantly in preventing mental retardation as compared to "old therapy"?

Whether the age at the first symptom, at diagnosis or whether plasma ammonia concentrations measured during the first work-up are of help for predicting a normal or compromised neuro-developmental outcome are presented elsewhere [2].

\section{Patients and methods}

A total of 134 questionnaires had been sent out to colleagues in regional hospitals of varying size as well as to specialised metabolic centres who had submitted samples for diagnosis or consulted us for patients with UCD at the Clinical Chemistry Central Laboratory of the University Hospital of Berne between 1975 and 1986. Out of 90 questionnaires returned, 88 contained sufficient information: 44 on patients with "old" therapy and 44 with "new therapy". The latter had been treated according to principles summarised elsewhere [1].

The procedures followed by the various hospitals probably reflect the reality one would face with a screening programme more closely than data from a selected patient population of major centres. There was no exclusion of patients who were symptomatic as neonates or who did not survive 28 days. Further details about the diagnoses are given elsewhere [2].

\section{Results}

Survival data are shown in Fig. 1. Survival is better in the "new" therapy group up to $2-3$ years of age; this is due mainly to the newborn group on "new" therapy where survival is significantly higher than with "old" therapy ( $P<0.045$, Fischers exact test), but not thereafter.

If the surviving patients are analysed, one realises that the rate of retarded patients is significantly higher with "new" therapy than with "old" therapy in the neonatal group $(P=0.0325)$. Age of first symptom $<28$ days: ten were retarded, 5 normal out of 15 surviving (and 13 dead) treated by "new" therapy, while with "old" therapy, the four surviving (22 dead) were normal at outcome. For the group with symptomatic presentation after the neonatal age, the difference is not statistically different (see [2] for details).

When discussing the question of screening, the age at which the first symptom was noticed is relevant. As shown in Fig. 2, 56\% of all the patients were symptomatic within 4 days of age and $67 \%$ within the 1 st week. 
Fig. 1 Survival curve (KaplanMeier) of patients with new therapy (lines) compared to old therapy (dotted lines). The outer thinner lines are the corresponding $95 \%$ confidence limits

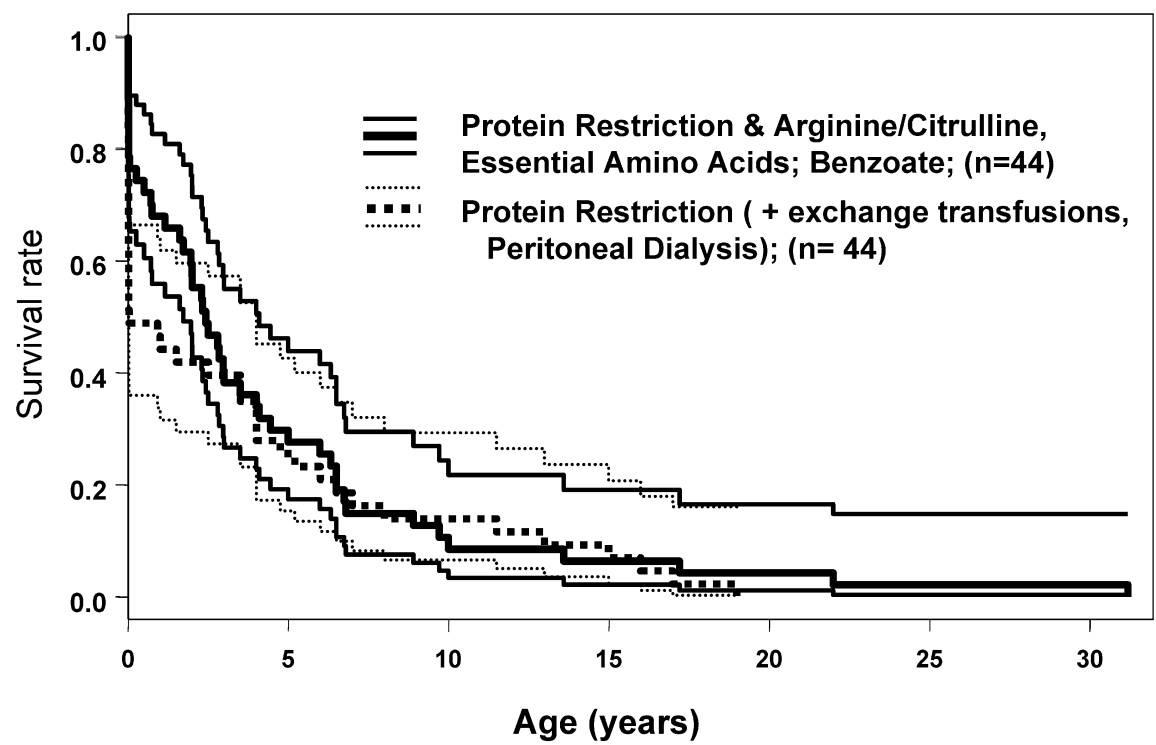

\section{Discussion}

The progress made in the treatment of UCDs has clearly improved the survival of patients during the first 2-3 years, but at the cost of more retarded patients. When excluding neonatal presentation, the survival figures presented here reflect the experience reported by others $[5,8,11]$. The high rate of retarded children is in accordance with the data of Maestri et al. [7] for argininosuccinic aciduria treated mostly with phenylbutyrate. The prognosis has been shown to be better in

Fig. 2 Age at which the first symptoms were observed, shown according to diagnosis. ( $A L$ argininosuccinate lyase deficiency (argininosuccinic aciduria), $A R G$ arginase 1 deficiency (argininaemia), $A S$ argininosuccinate synthetase deficiency (citrullinaemia type 1), CPS carbamoylphosphate synthetase deficiency, OTCm and OTCf ornithine transcarbamylase deficiency (male and female). Other: lysinuric protein intolerance, hyperammonaemiahyperornithinaemia-homocitrullinuria syndrome, N-acetylglutamate synthetase deficiency, and transient hyperammonaemia of the premature newborn) prospectively treated newborns; these were not included in this study [6].

UCDs are characterised by the fact that environmental factors like minor infections which are inevitable and excessive protein restriction lead to catabolism as does an excessive protein intake (sometimes found in puberty). Even if genotype/phenotype relationships can be shown, the interference by environmental factors is such in UCD that a high uncertainty for prognosis remains. These factors are often unpredictable and, in contrast to PKU, lead to major crises and irreversible damage.

With the current schedule of blood sampling for neonatal screening on the 3rd or 4th day of life, more than $50 \%$ of the symptomatic patients would be missed. Since at that age the mean ammonia level greatly exceeds $300 \mu \mathrm{mol} / 1$ in symptomatic patients, these will most likely be retarded $[2,13]$.

Prolongation of life as such is not a goal of medicine. Many parents, families and patients suffer from restrictions imposed by the stringent diet, the dependency of a metabolic centre for monitoring, the recurrent anxiety

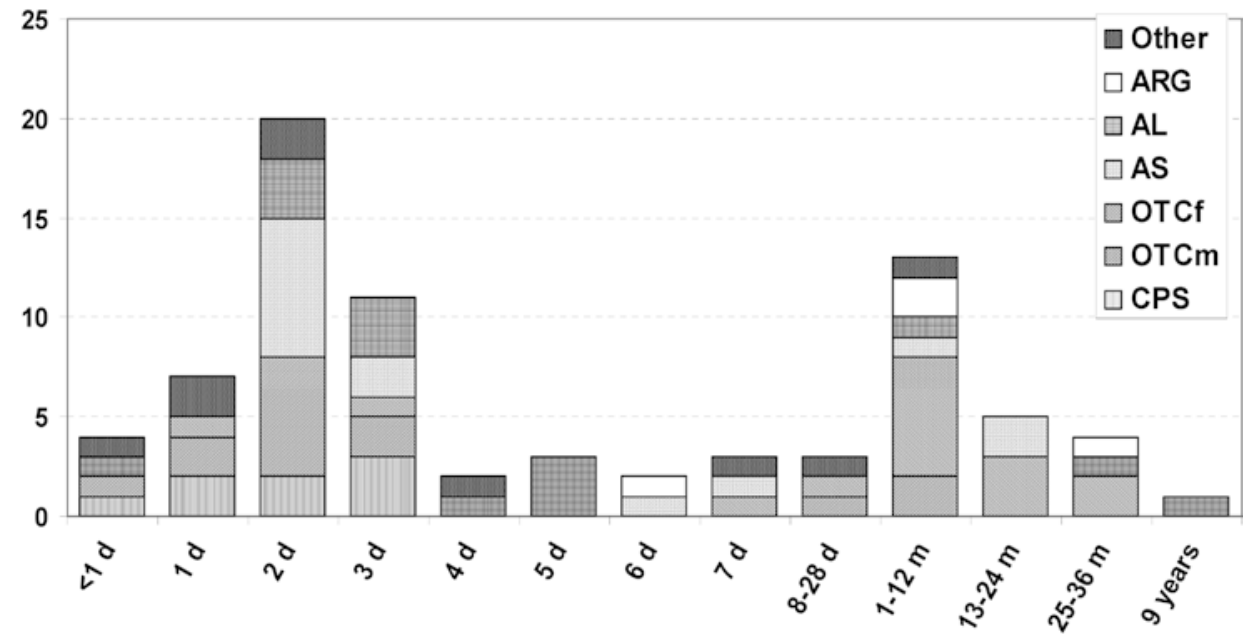


raised by crises, the many hospitalisations and especially from the disabilities of the patients. Since the present treatment appears not to be effective on long term, a population screening for UCD is, in our opinion, not indicated at present. The absence of a sufficiently dense referral organisation (not limited to a few major tertiary hospitals) with competent regional specialised centres for rapid work-up and diagnosis and metabolic crisis management, tight control of treatment and support to the parents is one of the major additional arguments against neonatal screening. One should be aware that a long delay in confirming (or excluding) the diagnosis contributes to the vulnerable child syndrome ("PKU anxiety syndrome) [12].

If a network of efficient diagnostic competence centres is installed and if the resources needed for treatment, parent support and counselling are guaranteed on a long-term basis, it can well be argued that even with a bad prognosis and absence of proven effective treatment, the offer of a tentative diagnosis can be a major benefit even if it is decided not to treat; knowing the cause of death of a child renders this tragedy more acceptable to a family and allows genetic counselling and prospective treatment if chosen by the parents. Such arguments would have to be included in the prenatal information and explained to the parents to allow them to refuse screening if this is not acceptable to them.

Technical problems still exist for the early detection of all UCDs or hyperammonaemic disorders. The sensitivity and specificity (tolerable false-positive rate) and choice of metabolite panel (e.g. high and low citrulline, arginine, lysine, alanine, orotate) is not clear. Prior to starting pilot studies, data are needed for comparing the plasma with red cell concentration of these metabolites under pathological conditions (at equilibrium in view of the highly variable haematocrit in newborns) and on the influence of the time of sampling after the end of the last feeding. In our experience, the influence of meals (amount of protein and post-prandial time delay of sampling) on citrulline would hamper the use of low citrulline for detecting mitochondrial UCDs. The pre-analytical instability of glutamine (glutamate and pyroglutamate formation) is well known. To restrict screening to a few of the UCDs or hyperammonaemic disorders bears the risk that neonatologists and paediatricians who are not aware of the whole complex spectrum of hyperammonaemic disorders might exclude those by falsely assuming that all have been excluded by the "metabolic screening".

For those patients who develop normally, the known complications and more predictable risks of liver transplantations and chronic immunosuppressive therapy (for the mitochondrial defects) should be weighed against the long-term risks of the treated UCD in each individual case. Assessing in vivo the residual activity of the urea cycle might be of help for this risk assessment [16].

As general knowledge about inborn errors has improved since the 1980s and the importance of diagnosing, installing rapidly an adequate treatment, monitoring and adapting it to the needs of the patients has been better recognised, further efforts must be made for continuous education (including small hospitals). The schematic application of therapeutic recommendations bears the danger of aminoacid imbalance and chronic catabolism by excessive supply of benzoate or phenylbutyrate and insufficient supplement of essential aminoacids.

\section{Conclusions}

We certainly need new therapeutic approaches and, for this, basic knowledge on the pathogenesis of irreversible damage to the brain by hyperammonaemic conditions. In practice, plasma ammonia (not capillary blood) should be assayed in sick newborns much more liberally $(24 \mathrm{~h} / 24 \mathrm{~h})$, e.g. whenever there is a sepsis work-up and in older patients with loss of appetite, vomiting and neurological symptoms. Plasma and a spot urine should be collected and the patient transferred without hesitation to a close centre with experience and competence in treating UCDs.

\section{References}

1. Bachmann C (1990) Urea cycle disorders. In: Fernandes J, Saudubray J, Tada K (eds) Inborn metabolic diseases: diagnosis and treatment. Springer, Berlin Heidelberg New York, pp 211-228

2. Bachmann C (2003) Outcome and survival of 88 patients with urea cycle disorders: a retrospective evaluation. Eur J Pediatr 162: 410-416

3. Bearn AG, Cusworth DC, Dean G, Frézal JP, Neifakh SA, Scheinberg IH, Scriver CR, Szeinberg A (1968) Screening for inborn errors of metabolism. WHO Tech Rep Ser 401: 1-57

4. Bickel H, Bachmann C, Beckers R, Brandt NJ, Clayton BE, Corrado G, Feingold HJ, Giardini O, Hammersen G, Schoenberg D (1981) Neonatal mass screening for metabolic disorders. Eur J Pediatr 137: 133-139

5. Brusilow SW, Horwich AL (2001) Urea cycle enzymes. In: Scriver CR, Beaudet AL, Sly WS, Valle D (eds) The metabolic and molecular basis of inherited disease, vol II. McGraw-Hill, New York, pp 1909-1963

6. Maestri NE, Hauser ER, Bartholomew D, Brusilow SW (1991) Prospective treatment of urea cycle disorders. J Pediatr 119: 923-928

7. Maestri NE, Clissold DB, Brusilow SW (1995) Long-term survival of patients with argininosuccinate synthetase deficiency. J Pediatr 127: 929-935

8. Maestri NE, Brusilow SW, Clissold DB, Bassett SS (1996) Long-term treatment of girls with ornithine transcarbamylase deficiency. N Engl J Med 335: 855-859

9. Msall M, Batshaw ML, Suss R, Brusilow SW, Mellits ED (1984) Neurologic outcome in children with inborn errors of urea synthesis. Outcome of urea-cycle enzymopathies. N Engl J Med 310: 1500-1505

10. Pollitt RJ, Green A, McCabe CJ, Booth A, Cooper NJ, Leonard JV, Nicholl J, Nicholson P, Tunaley JR, Virdi NK (1997) Neonatal screening for inborn errors of metabolism: cost, yield and outcome. Health Technol Assess 1: 1-202

11. Saudubray JM, Touati G, Delonlay P, Jouvet P, Narcy C, Laurent J, Rabier D, Kamoun P, Jan D, Revillon Y (1999) Liver transplantation in urea cycle disorders. Eur J Pediatr 158[Suppl 2]: S55-S59 
12. Tluczek A, Mischler EH, Farrell PM, Fost N, Peterson NM, Carey P, Bruns WT, McCarthy C (1992) Parents' knowledge of neonatal screening and response to false-positive cystic fibrosis testing. J Dev Behav Pediatr 13: 181-186

13. Uchino T, Endo F, Matsuda I (1998) Neurodevelopmental outcome of long-term therapy of urea cycle disorders in Japan. J Inherit Metab Dis 21[Suppl 1]: 151-159

14. Wiech N, Clissold D, MacArthur R (1997) Safety and efficacy of buphenyl (sodium phenylbutyrate) tablet and powder (abstract). Advances in Inherited Urea Cycle Disorders, Vienna, Austria, pp 25

15. Wilson JMG, Jungner G (1968) Principles and practice of screening for disease. Public Health Papers WHO 34: 26-39

16. Yudkoff M, Daikhin Y, Ye X, Wilson JM, Batshaw ML (1998) In vivo measurement of ureagenesis with stable isotopes. J Inherit Metab Dis 21: 21-29 